\title{
LA-UR-21-24874
}

Approved for public release; distribution is unlimited.

Title: iFP simulations for Omega experiments studying implosions about the cryogenic vapor pressure of $0.3 \mathrm{mg} / \mathrm{cc}$

Author(s):

Taitano, William

Kabadi, Neel

Bose, Arijit

Intended for: $\quad$ Presentation for external collaborators

Issued: 
Disclaimer:

Los Alamos National Laboratory, an affirmative action/equal opportunity employer, is operated by Triad National Security, LLC for the National Nuclear Security Administration of U.S. Department of Energy under contract 89233218CNA000001. By approving this article, the publisher recognizes that the U.S. Government retains nonexclusive, royalty-free license to publish or reproduce the published form of this contribution, or to allow others to do so, for U.S. Government purposes. Los Alamos National Laboratory requests that the publisher identify this article as work performed under the auspices of the U.S. Department of Energy. Los Alamos National Laboratory strongly supports academic freedom and a researcher's right to publish; as an institution, however, the Laboratory does not endorse the viewpoint of a publication or guarantee its technical correctness. 


\section{iFP simulations for Omega experiments studying implosions about the cryogenic vapor pressure of $0.3 \mathrm{mg} / \mathrm{cc}$}

Arijit Bose (UofD)

Neel Kabadi (MIT)

William Taitano (LANL) 


\section{Outline}

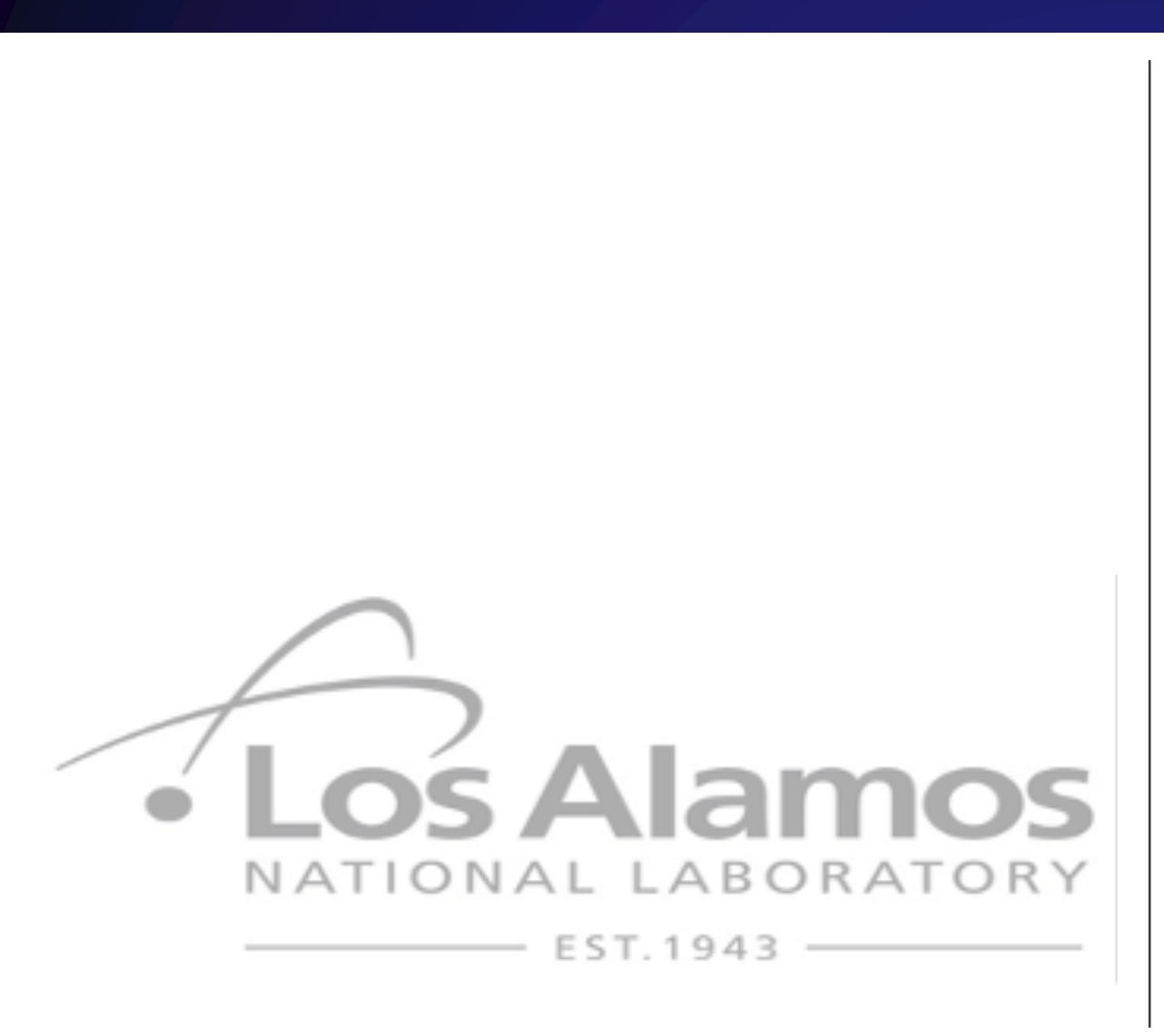

- Brief summary of iFP

- Model: Hybrid ion-VFP and fluid electrons

- Nonlinearly implicit solver

- Adaptive and conservative phase-space grid

- MIT exploding pusher experiments

- IC and BC

- Results and discussions

- YOC in good agreement

- Inferred temperature also closer to experiment 


\section{IFP: A hybrid 1D2V VFP code for spherical implosion simulations of ICF capsules}

- Hybrid VFP ion (arbitrary species) and fluid electron with quasi-neutrality and ambipolarity

$$
\begin{gathered}
\partial_{t} f_{\alpha}+\nabla_{x} \cdot\left(\vec{v} f_{\alpha}\right)+\nabla_{v} \cdot\left(\vec{a} f_{\alpha}\right)=\nabla_{v} \cdot\left[\overline{\bar{D}}_{\beta} \cdot \nabla_{v} f_{\alpha}-\vec{A}_{\beta} f_{\alpha}\right] \\
\frac{3}{2} \partial_{t} P_{e}+\frac{5}{2} \nabla_{x} \cdot\left(\vec{u}_{e} P_{e}\right)-\vec{u}_{e} \cdot \nabla_{x} P_{e}=\sum_{\alpha}^{N_{s}} W_{e \alpha} \\
n_{e}=\sum_{\alpha}^{N_{s}} q_{\alpha} n_{\alpha} / q_{e} \quad \vec{u}_{e}=\sum_{\alpha}^{N_{s}} n_{\alpha} \vec{u}_{\alpha} / q_{e} n_{e}
\end{gathered}
$$

- 1D2V spherical radial and cylindrical velocity space

- Electrostatic (Ohm's law)

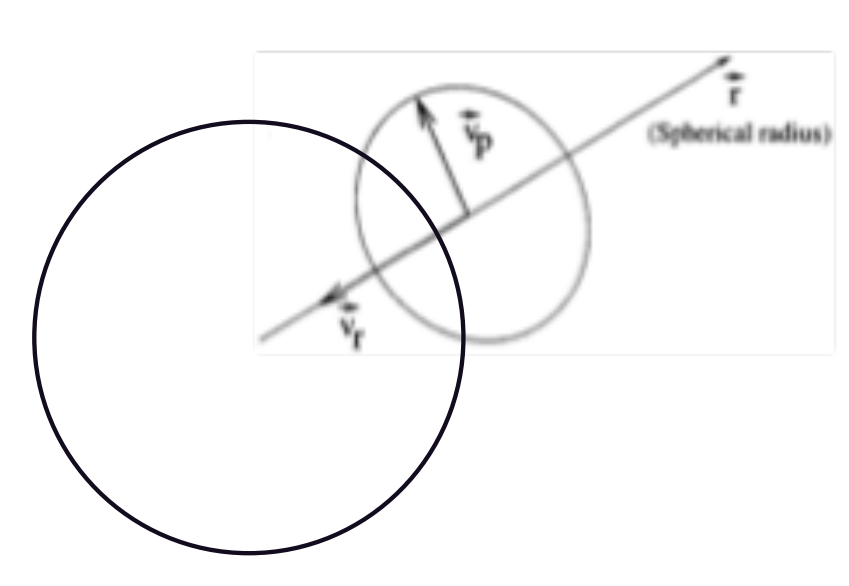




\section{Nonlinearly implicit time-stepping and adaptive phase-space grid}

- Nonlinearly implicit Solver:

- $\Delta t_{\text {exp }} \sim O\left(10^{-12} n s\right)$ (dynamically irrelevant)

- $\tau_{d y n} \sim O\left(10^{-3} n s\right)$

- Adaptive and conservative phase-space grid

- Static uniform grid : $O\left(10^{12}\right)$ unknowns in $1 \mathrm{D} 2 \mathrm{~V}$

- Adaptive grid : $O\left(10^{5}\right)$ unknowns
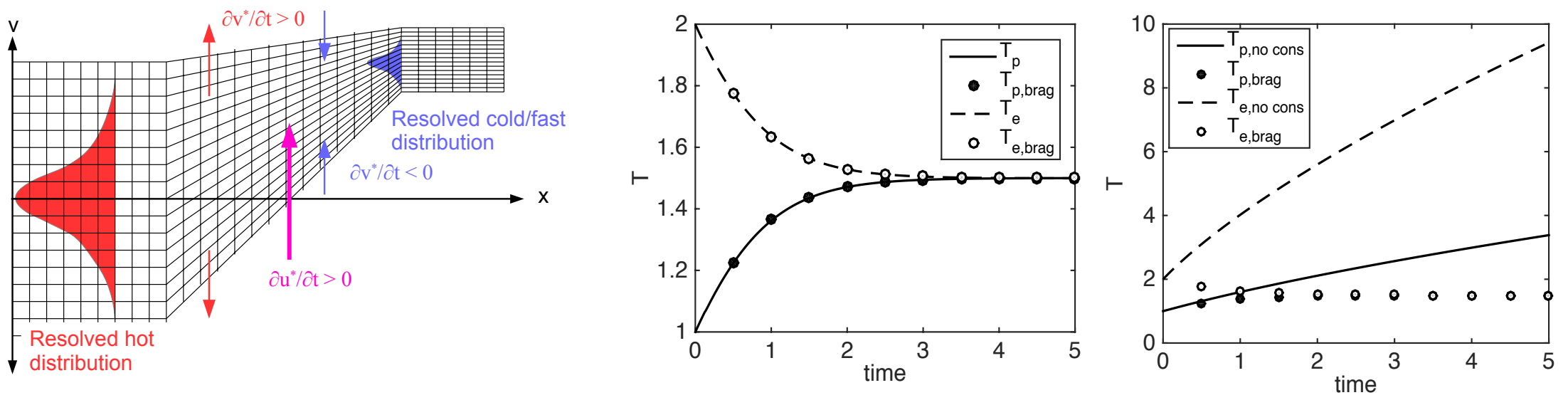


\section{The implementation is massively parallel and scalable as well}

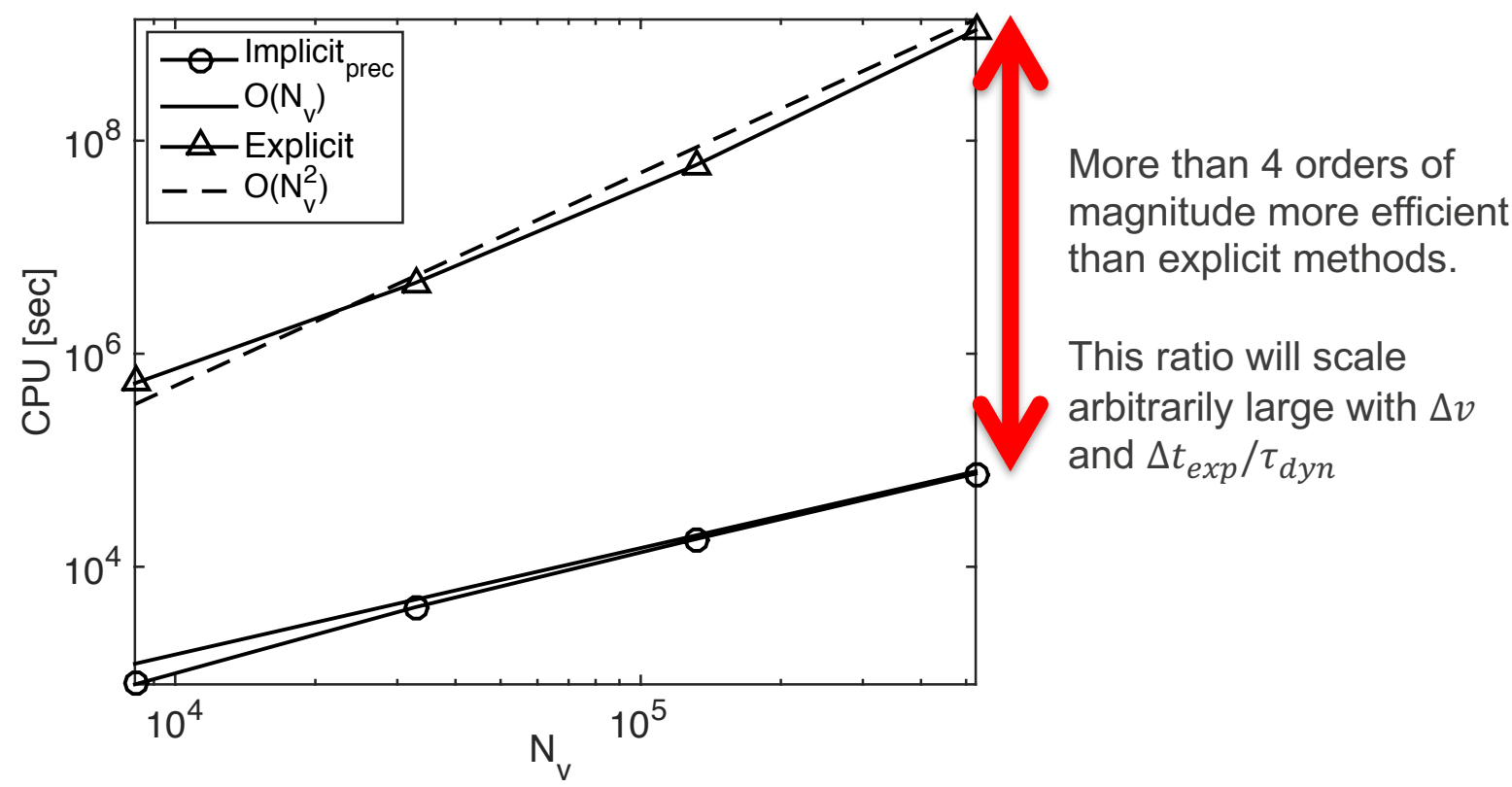

W.T. Taitano et al., JCP 297 (2015) 
iFP has been benchmarked rigorously against a broad suite of analytical and semi-analytical theory

- Correct self-similar solution ${ }^{1}$ obtained for $t \gg \tau_{\text {col }}$

- Test of implicit solver with $\Delta t=4 \times 10^{4} \tau_{\text {col }}$

- Successfully benchmarked against VPIC2
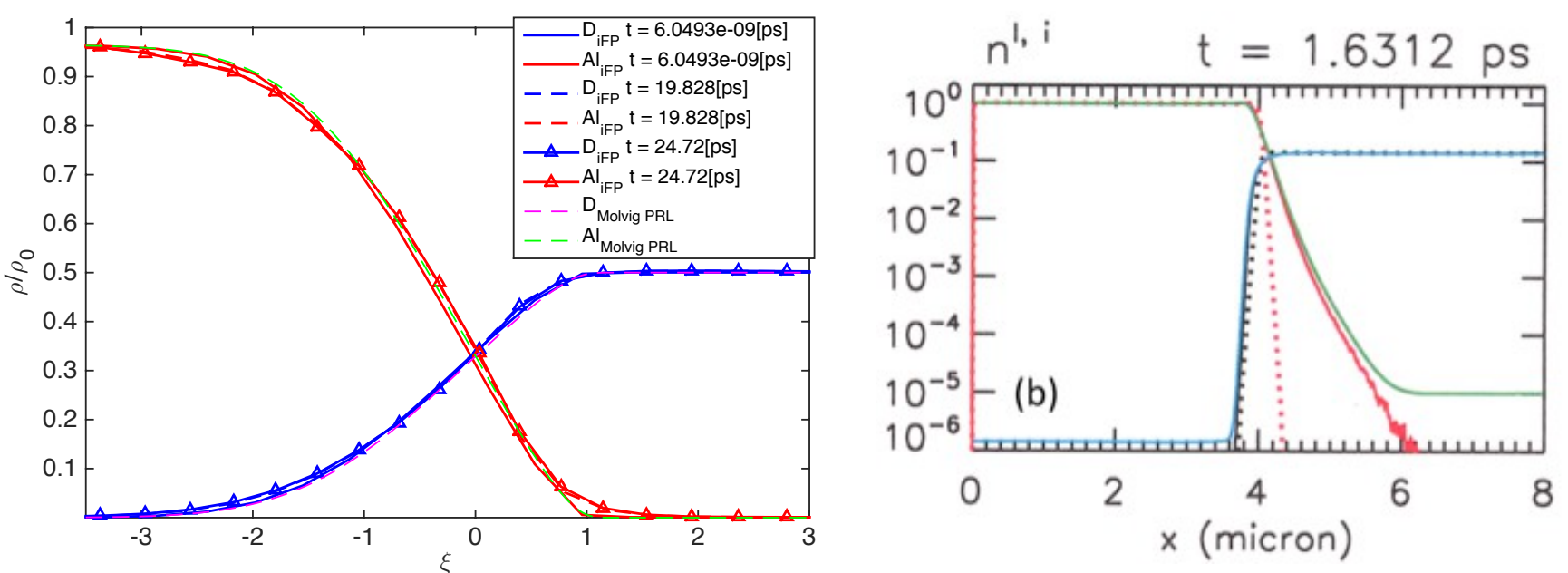

1. K. Molvig et al., PRL 113 (2014)

2. Yin et al, Phys. Plasmas, 23, 112302 (2016) 


\section{M=1.5 Multispecies Shock (fluid regime)}
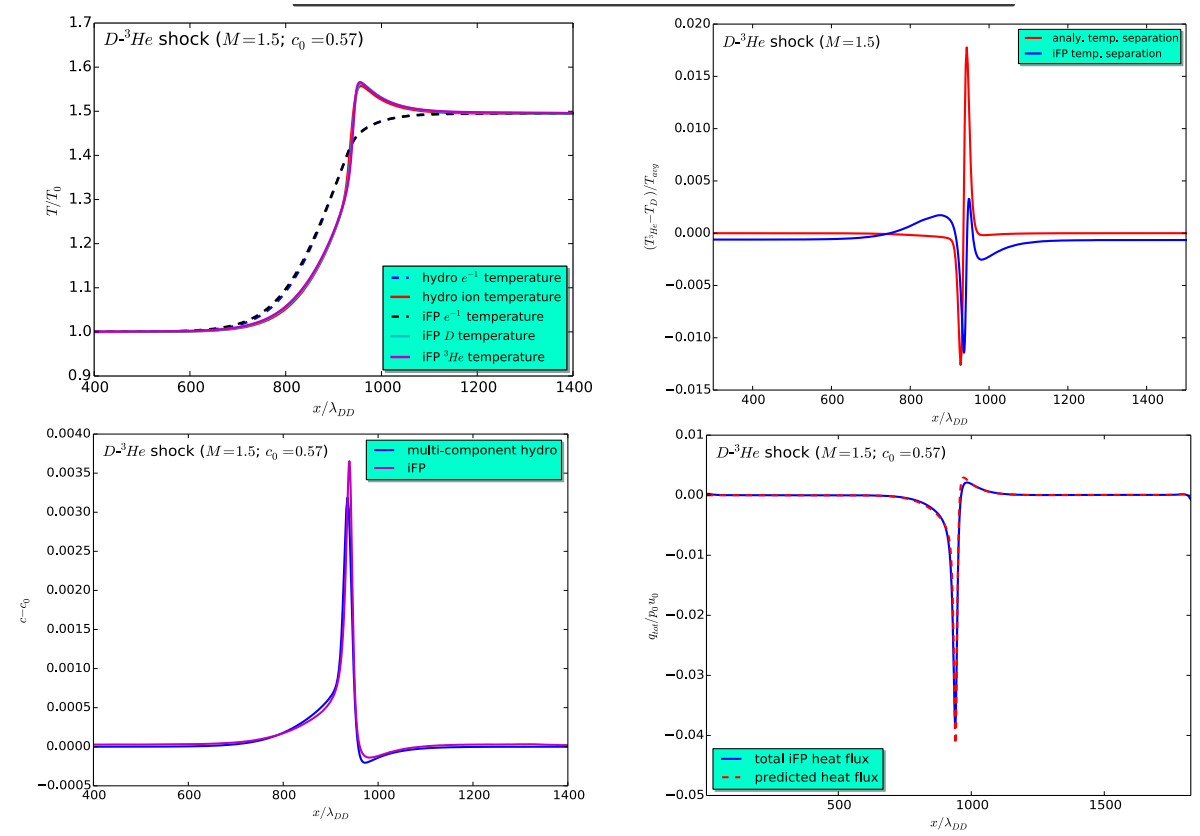

B. Keenan et al., Phys. Rev. E, 96 (2018) 


\section{M=5 Multispecies Shock (fluid regime)}

Single-species (verification)

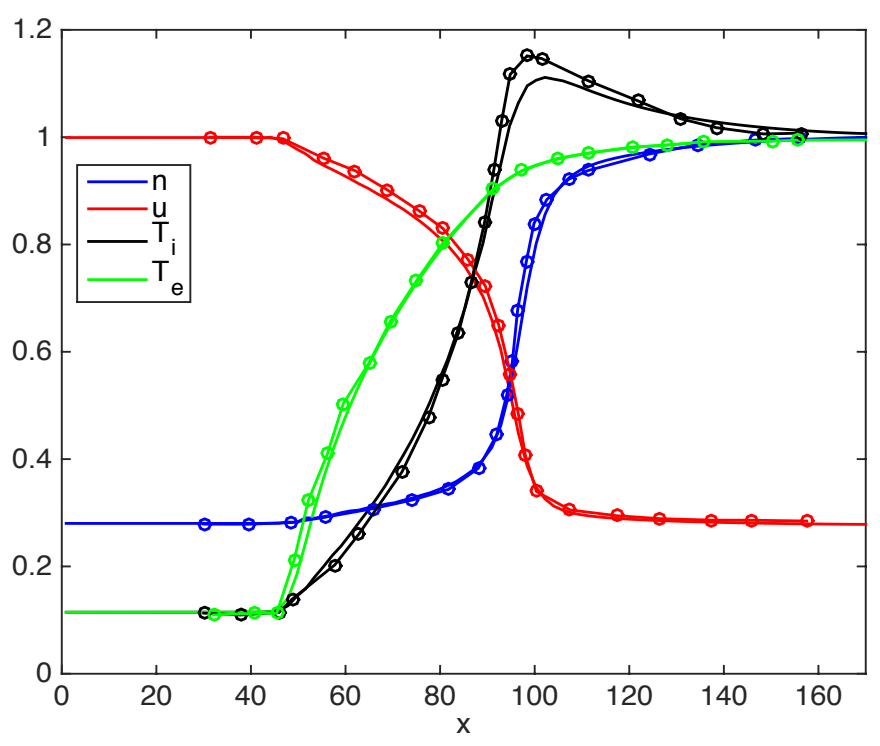

Vidal et al., Phys. Fluids B, 5 (9) (1993)

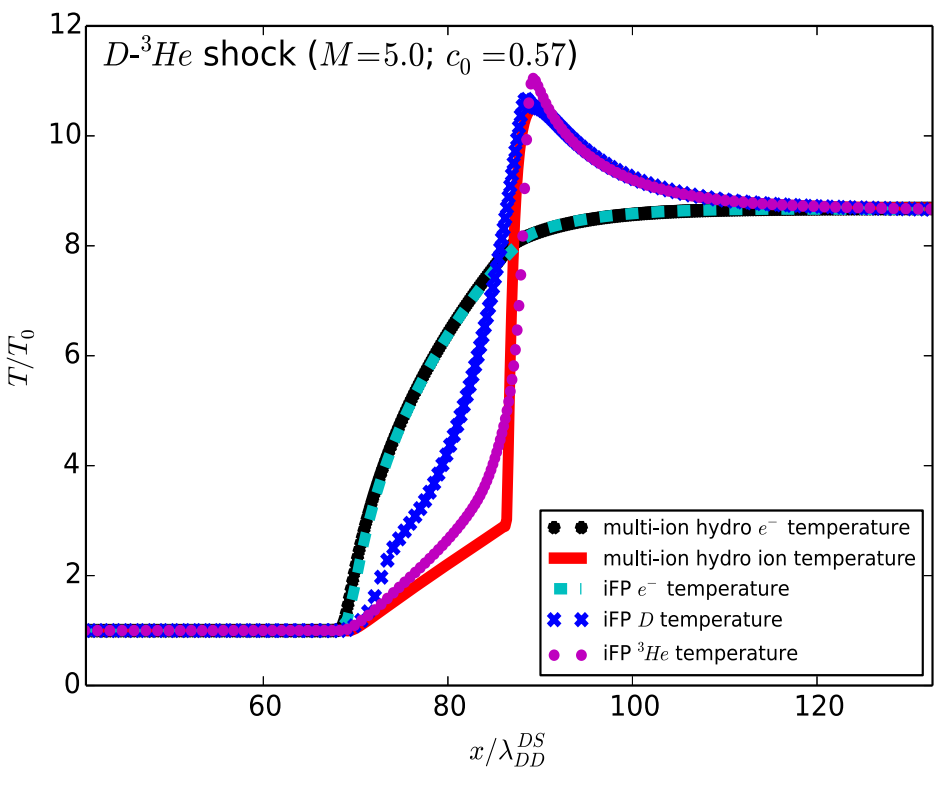

Keenan et al., PRE, 96 (2017) 


\section{Spherical Guderley problem}
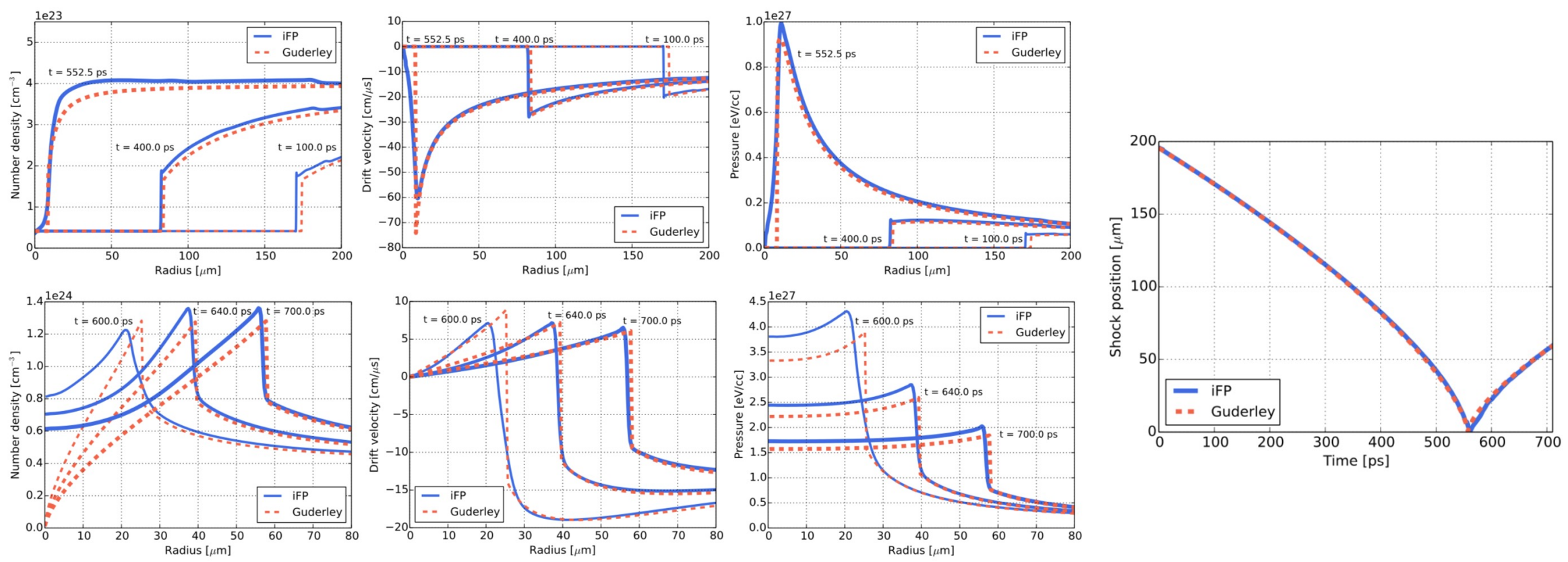

Taitano et al., Comp. Phys. Comm., 263 (2021) 


\section{are used to initialize and drive iFP simulations}
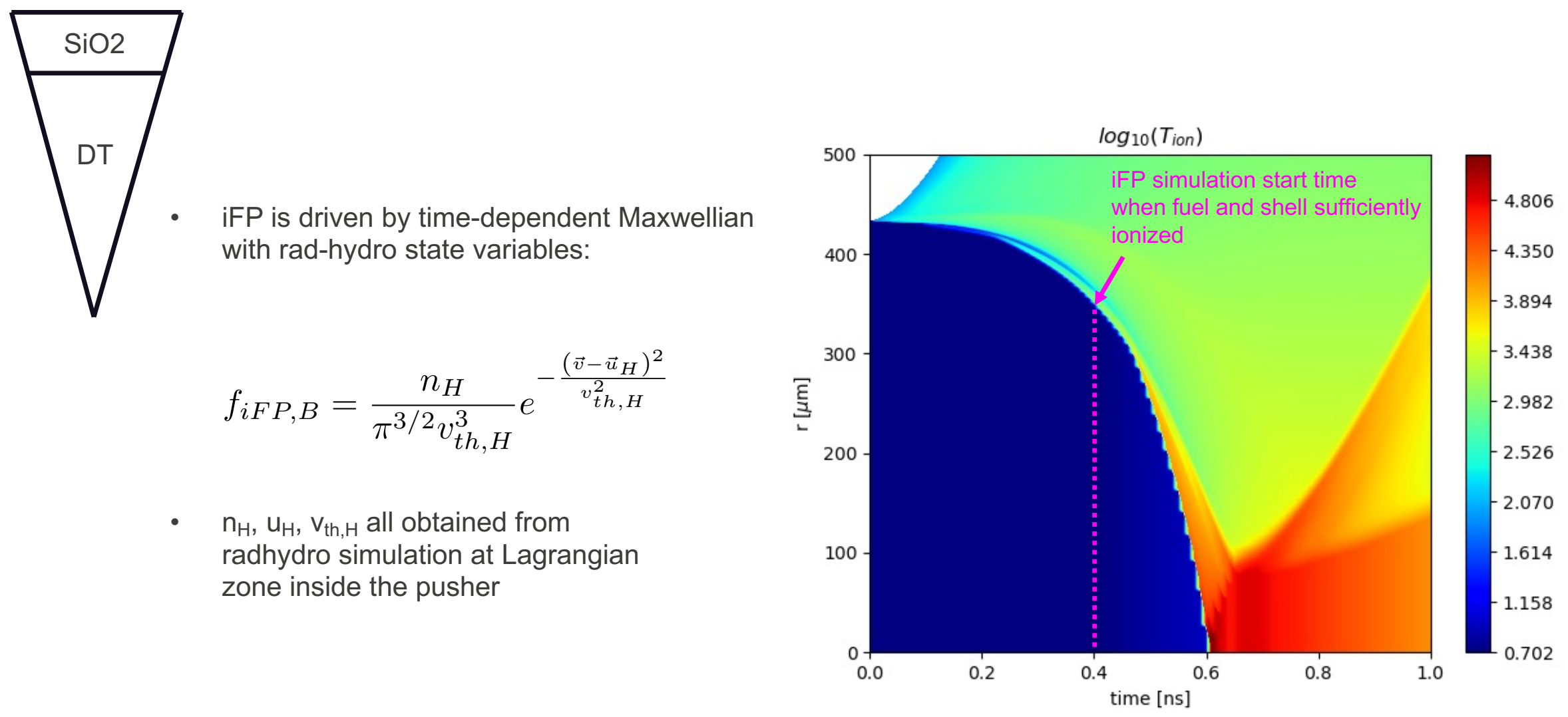
Observations: Kinetically enhance pusher mix, as well as fuel ballistically streaming out of the core
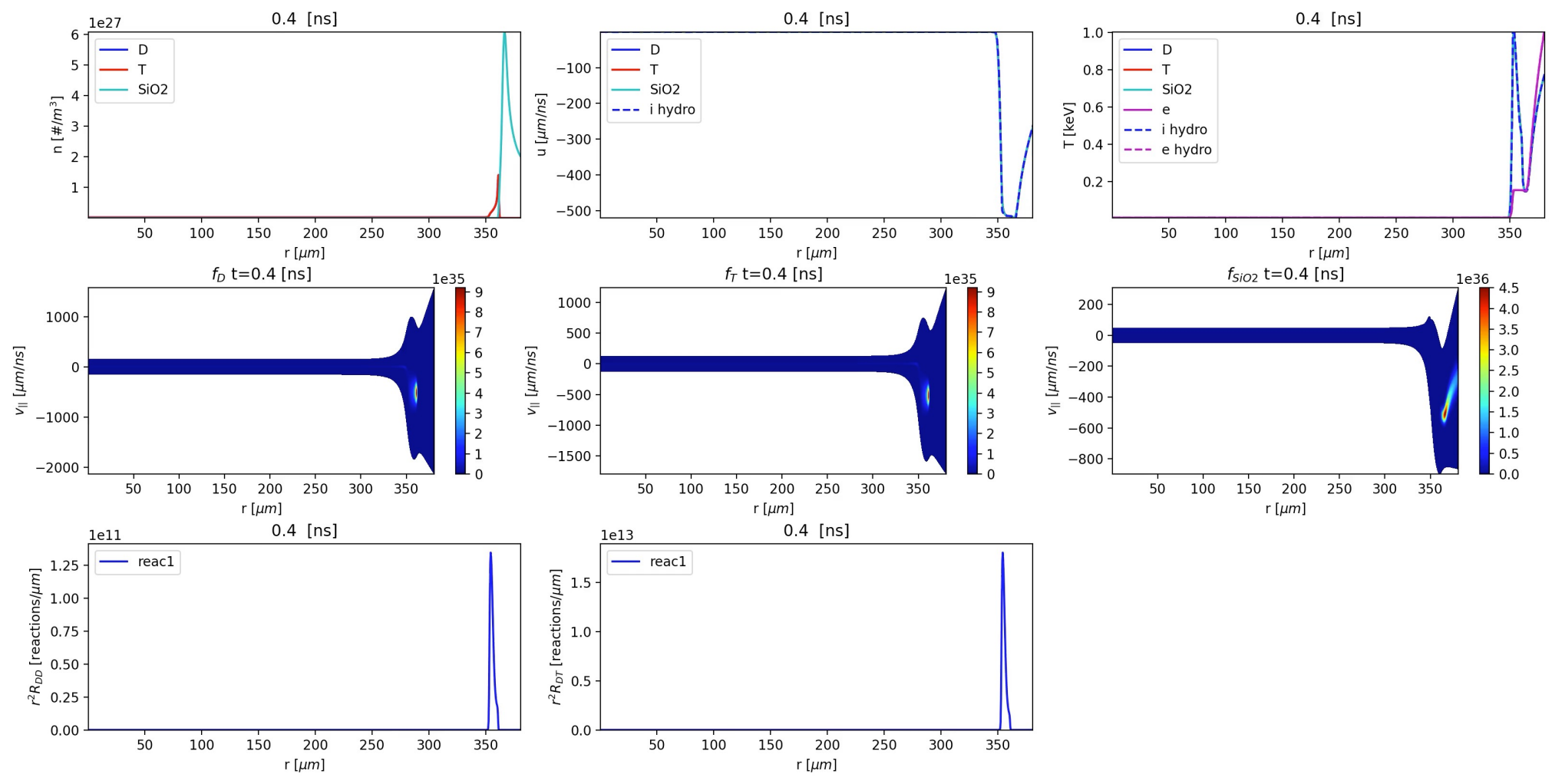
Significant mix of glass shell into core at bang time. lim effects in X-ray image from rad-hydro is not present
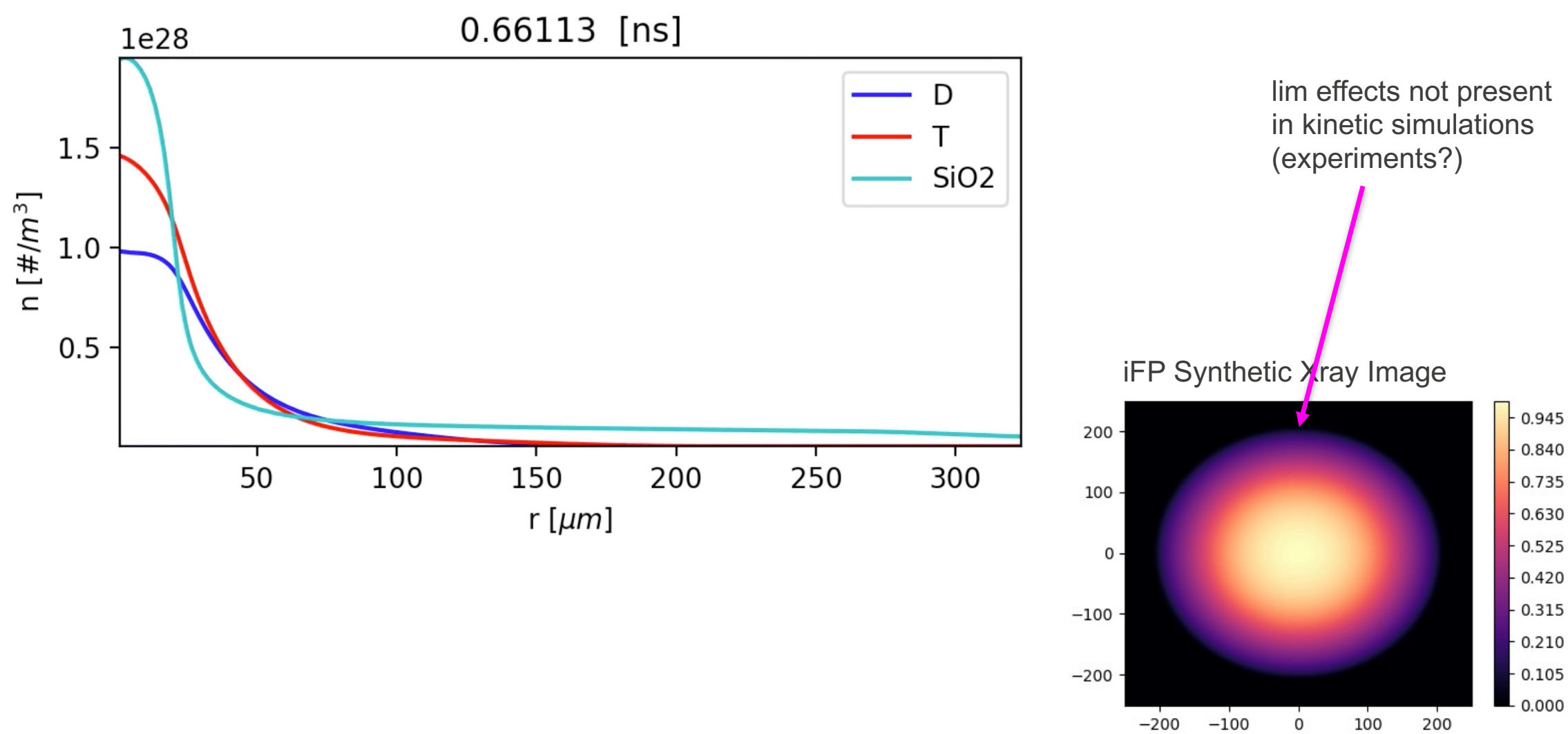


\section{Good agreement in YOC up until the lowest fill pressure}

DTn and DDn YOC in 50:50 DT exploding pushers

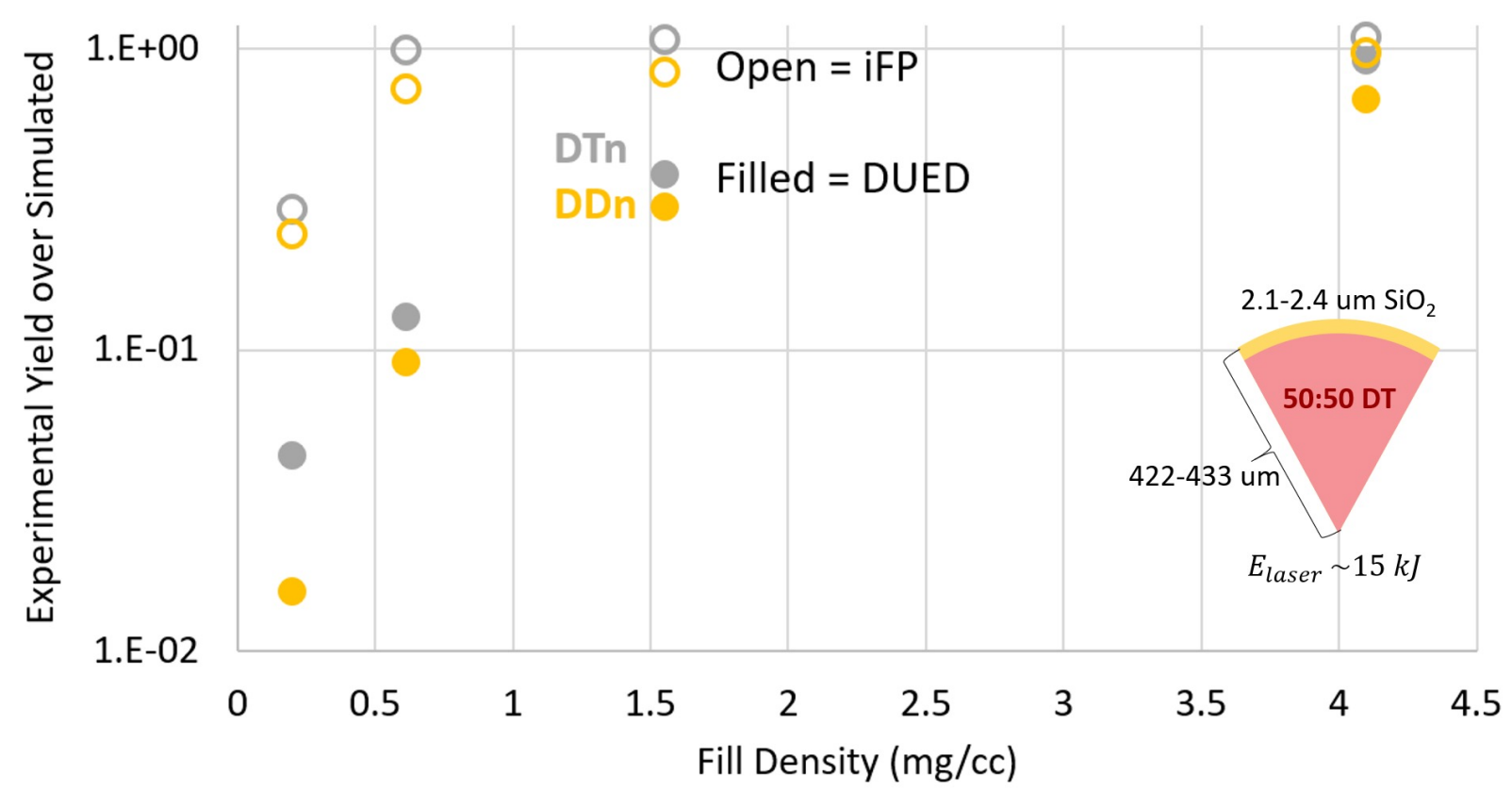


Inferred (neutron spectrum) ion temperatures in closer agreement with experiments, relative to LILAC (driver for iFP), but DUED performs better at higher fill

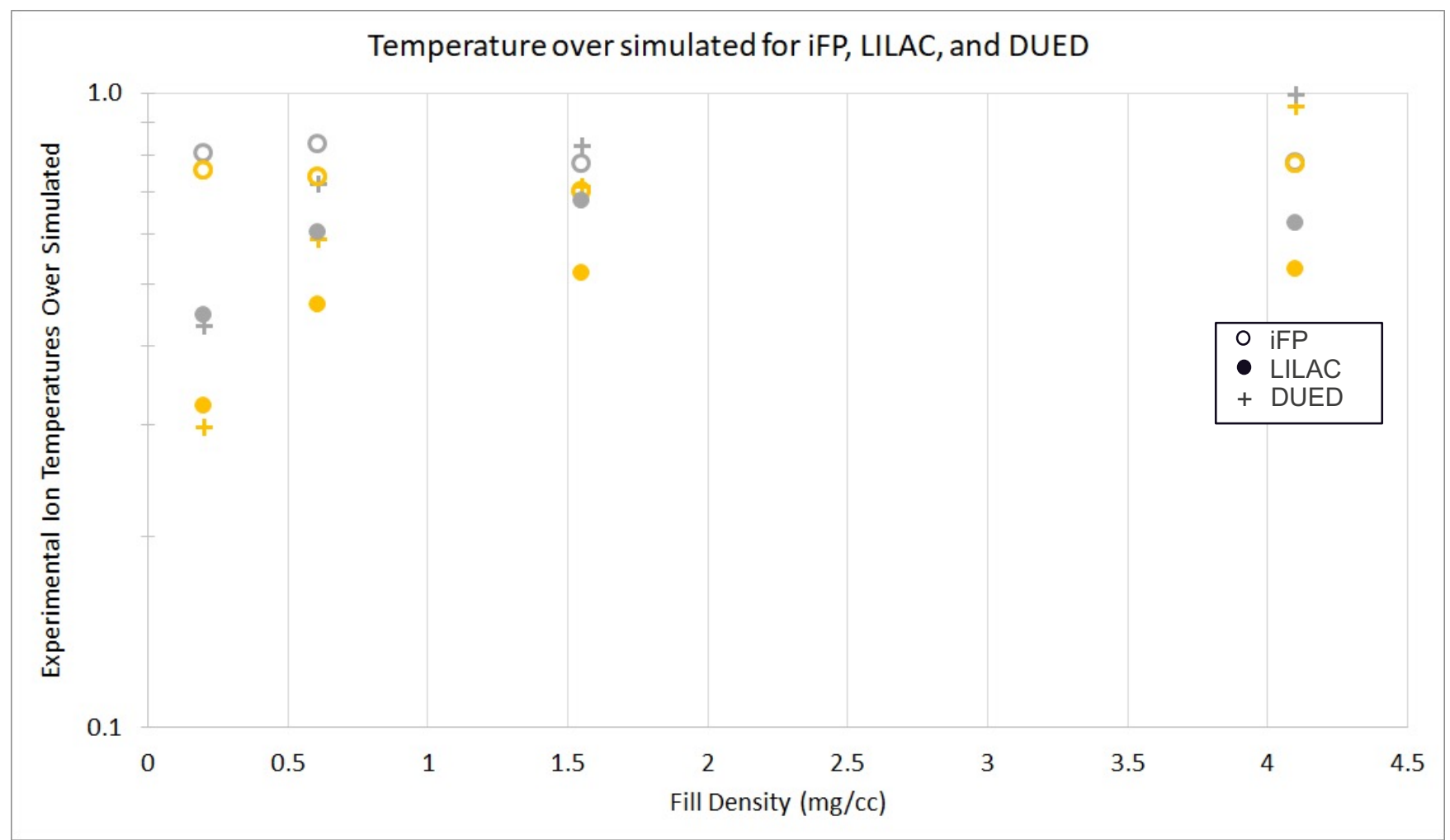




\section{Immediate and future work}

- Isolation of dominant physics that iFP is capturing correctly:

- Interface mixing

- Tail depletion

- Increased heat capacity due to shell mixing

- Missing physics in current simulation:

- Kinetic electrons:

- Nonlocal electron heat-transport and kinetic instabilities role in implosion dynamics?

- Capability currently being tested in iFP with shot 86639. Numerical issues are being ironed out.

- Electron EOS:

- For higher fill pressures, could play a role particularly in pusher.

- Self-consistent radiation drive:

- Currently, testing gray radiation diffusion (for indirect drive).

- Laser driver for direct drive will be more relevant for this collaboration. 